\title{
Anomalous Hall Effect in Ferromagnetic Semiconductors in the Hopping Transport Regime
}

\author{
A.A. Burkov and Leon Balents \\ Department of Physics, University of California, Santa Barbara, California 93106, USA
}

(Dated: November 2, 2018)

\begin{abstract}
We present a theory of the Anomalous Hall Effect (AHE) in ferromagnetic (Ga,Mn)As in the regime when conduction is due to phonon-assisted hopping of holes between localized states in the impurity band. We show that the microscopic origin of the anomalous Hall conductivity in this system can be attributed to a phase that a hole gains when hopping around closed-loop paths in the presence of spin-orbit interactions and background magnetization of the localized Mn moments. Mapping the problem to a random resistor network, we derive an analytic expression for the macroscopic anomalous Hall conductivity $\sigma_{x y}^{A H}$. We show that $\sigma_{x y}^{A H}$ is proportional to the first derivative of the density of states $\varrho(\epsilon)$ and thus can be expected to change sign as a function of impurity band filling. We also show that $\sigma_{x y}^{A H}$ depends on temperature as the longitudinal conductivity $\sigma_{x x}$ within logarithmic accuracy.

PACS numbers: 75.50.Pp,73.50.Jt,72.20.Ee
\end{abstract}

Diluted magnetic semiconductors (DMS) are very interesting new materials, still not understood in full detail. Although it is well accepted that the origin of ferromagnetism in $\mathrm{Ga}_{1-x} \mathrm{Mn}_{x} \mathrm{As}$, the most thoroughly studied DMS, is a hole-mediated effective interaction between the localized $S=5 / 2 \mathrm{Mn}$ spins, the appropriate description of this interaction, and a closely related question of the nature of hole carriers, are still controversial issues. Samples with the highest Curie temperatures show metallic behavior 11 and are well described by the valenceband hole-fluid model 22. On the other hand, for low Mn concentrations, i.e. when $x$ is less than about 0.03 , $(\mathrm{Ga}, \mathrm{Mn})$ As is insulating and the hole transport likely occurs in the Mn impurity band 3]. The low-temperature conduction in this case is by phonon-assisted hopping.

Anomalous Hall Effect (AHE) has been an important characterization tool for itinerant ferromagnets [4, 5, 6 ] and played an important role in the experimental study of DMS ferromagnetism as well [7]. Experimentally AHE is manifested as an additional term in the Hall resistivity of the sample, usually assumed to be proportional to the magnetization:

$$
\varrho_{x y}=R_{0} B+R_{s} M .
$$

Standard theories of AHE in metallic ferromagnets [4, 6] attribute it to the modification of impurity scattering in the presence of spin-orbit interactions. Recently, however, it was realized that a purely geometrical mechanism of AHE is possible [8, 9, 10, 11]. In particular, a $\mathbf{k}$-space Berry phase theory of AHE 8 has been proposed, and applied specifically to $(\mathrm{Ga}, \mathrm{Mn}) \mathrm{As}$ in the metallic transport regime. This theory is very successful in describing AHE in metallic $(\mathrm{Ga}, \mathrm{Mn}) \mathrm{As}$, and we believe that the Berry-phase mechanism of AHE should be relevant in general for all itinerant ferromagnets.

In this Letter we propose an analogous geometricalphase theory of AHE in $(\mathrm{Ga}, \mathrm{Mn}) \mathrm{As}$, but in the case when conduction is by hopping between strongly localized states in the impurity band, rather than through extended valence-band states. Our approach is based on a modified Holstein's theory of the Hall effect in hopping conduction 12] (for related work on AHE in colossal magnetoresistance manganites see [10]).

We start from a model of spin-3/2 hole carriers, hopping between randomly distributed localizing centers (Mn acceptors) in the mean spin-splitting field of $S=5 / 2$ Mn local moments. The corresponding Hamiltonian is:

$$
H_{h}=\sum_{i \alpha} \epsilon_{i} c_{i \alpha}^{\dagger} c_{i \alpha}-\sum_{i \alpha j \beta} t_{i \alpha j \beta} c_{i \alpha}^{\dagger} c_{j \beta}-\sum_{i \alpha \beta} \mathbf{h} \cdot \boldsymbol{\tau}_{\alpha \beta} c_{i \alpha}^{\dagger} c_{i \beta} .
$$

Here $i, j$ label localized hole states at Mn acceptor sites, $\alpha, \beta=3 / 2, \ldots,-3 / 2$ are spin- $3 / 2$ indices, $\epsilon_{i}$ are random on-site potentials, which model the random potentials localized holes feel due to Coulomb interactions with nearby charged Mn acceptors and As antisite defects, $\mathbf{h}$ is the spin-splitting mean field due the p-d exchange interaction between the hole- and localized Mn-spins, $\boldsymbol{\tau}$ is the spin-3/2 operator and $t_{i \alpha j \beta}$ is a spin-dependent hopping amplitude which we assume to be of the following form:

$$
t_{i \alpha j \beta}=\mathcal{R}_{i j} \hat{t} \mathcal{R}_{i j}^{\dagger} e^{i A_{i j}-r_{i j} / \xi},
$$

where $\xi$ is the localization length and $A_{i j}=(e / 2 c) \mathbf{B}$. $\left(\mathbf{r}_{i} \times \mathbf{r}_{j}\right)$ is a phase factor due to the external magnetic field $\mathbf{B}$ (we use $\hbar=1$ units). In most of the following we will omit this factor. $\hat{t}=\operatorname{diag}\left(t_{3 / 2}, t_{1 / 2}, t_{1 / 2}, t_{3 / 2}\right)$ is a diagonal hopping matrix in the case when $\hat{r}_{i j}$ is along the spin quantization direction and $\mathcal{R}_{i j}$ rotates the spin quantization axis from $\hat{z}$ to $\hat{r}_{i j}$. The hopping parameters $t_{3 / 2}$ and $t_{1 / 2}$ can be calculated microscopically [13]. The fact that $t_{3 / 2} \neq t_{1 / 2}$ is a direct consequence of the spinorbit interactions.

We assume that the Fermi energy lies in the region where all states are strongly localized and thus the only 
possible conduction mechanism is through the phononassisted hopping. Thus hole-phonon interaction must be explicitly included in the model:

$$
\begin{aligned}
& H_{h-p h}=\sum_{i \alpha \mathbf{q}} v_{\mathbf{q}}^{i}\left(b_{\mathbf{q}}+b_{-\mathbf{q}}^{\dagger}\right) c_{i \alpha}^{\dagger} c_{i \alpha} \\
& H_{p h}=\sum_{\mathbf{q}} \omega_{\mathbf{q}} b_{\mathbf{q}}^{\dagger} b_{\mathbf{q}} .
\end{aligned}
$$

The full Hamiltonian is then given by

$$
H=H_{h}+H_{h-p h}+H_{p h}
$$

Local transport properties of the system described by Eq. (5) can be most conveniently calculated using a combination of the usual linear-response theory and a perturbation expansion in powers of $t_{i \alpha j \beta} /\left|\epsilon_{i}-\epsilon_{j}\right|$, which is justified by our assumption that all states are localized [14, 15]. Holstein [12] was first to realize that to capture the (ordinary) Hall effect under hopping conduction conditions, one needs to consider processes where amplitudes of direct and indirect (through a third site) hops between two sites interfere. Such processes appear at third order in the perturbation theory in $t_{i \alpha j \beta} /\left|\epsilon_{i}-\epsilon_{j}\right|$, while the longitudinal hopping conductivity can be described by second-order terms. To capture AHE, one needs to consider third-order terms as well. Evaluating the linear response expression for the current $I_{i j}$ between two localized states [15], including terms of up to third order in the hopping amplitudes and up to second order in hole-phonon interaction, one obtains:

$$
I_{i j}=G_{i j} V_{i j}+\sum_{k} F_{i j k}\left(V_{i k}+V_{j k}\right) .
$$

Here $V_{i j}=V_{i}-V_{j}$ is the electrochemical potential difference between the sites $i$ and $j . G_{i j}$ is an effective conductance between a pair of sites, which at low temperatures, such that $\left|\epsilon_{i}-\epsilon_{j}\right| / T \gg 1$, is given by:

$$
G_{i j}=\frac{e^{2} \gamma_{0}}{T} \sum_{\alpha \beta}\left|t_{i \alpha j \beta}\right|^{2} e^{-\epsilon_{i \alpha j \beta} / T} .
$$

Here $\gamma_{0}$ is a constant, proportional to the phonon density of states and the square of the hole-phonon interaction matrix element, and we have introduced the following notation:

$$
\begin{aligned}
& \epsilon_{i \alpha j \beta}=\frac{1}{2}\left(\left|\epsilon_{i \alpha}\right|+\left|\epsilon_{j \beta}\right|+\left|\epsilon_{i \alpha}-\epsilon_{j \beta}\right|\right), \\
& \epsilon_{i \alpha}=\epsilon_{i}-h \tau_{\alpha \alpha}^{z} .
\end{aligned}
$$

We have assumed in the above that $\mathbf{h}=h \hat{z}$. Henceforth we will also assume that $h$ is sufficiently small, so that we can restrict ourselves to linear response effects with respect to $h$. In particular this means that we disregard the influence of spin splitting on the longitudinal conduction. We expect this to be a reasonable approximation at low
Mn concentrations, when the magnetization is relatively small.

The first term in Eq. (6) thus represents the usual mapping of the hopping conduction problem onto a random resistor network 16, 17, 18]. The second term in Eq. (6) contains the physics responsible for AHE. The quantity $F_{i j k}$ is given, in the low temperature limit, by the following expression:

$$
\begin{aligned}
& F_{i j k}=\frac{e^{2} \gamma_{0}^{2}}{8 T} \sum_{\alpha \beta \gamma} \Im\left(t_{i \alpha j \beta} t_{j \beta k \gamma} t_{k \gamma i \alpha}\right)\left[e^{\left(\left|\epsilon_{k \gamma}\right|-\epsilon_{i \alpha k \gamma}-\epsilon_{k \gamma j \beta}\right) / T}\right. \\
& \left.+e^{\left(\left|\epsilon_{i \alpha}\right|-\epsilon_{k \gamma i \alpha}-\epsilon_{i \alpha j \beta}\right) / T}+e^{\left(\left|\epsilon_{j \beta}\right|-\epsilon_{i \alpha j \beta}-\epsilon_{j \beta k \gamma}\right) / T}\right]
\end{aligned}
$$

Let us discuss the physical meaning of this equation. The process, that leads to the appearance of the term, proportional to $F_{i j k}$, in the expectation value of the current between sites $i$ and $j$, is a quantum interference process, where probability amplitudes of a direct hop between any two sites in a triad $(i, j, k)$ and an indirect hop (i.e. through the third site) interfere. Such a process requires the participation of at least two phonons, hence the factor $\gamma_{0}^{2}$. The crucial component of $F_{i j k}$ is the imaginary part of the product of three complex spin-dependent hopping amplitudes taken along the sides of the triangle formed by the sites $i, j, k$. It is directly related to the phase that a hole gains while hopping along a closed-loop three-site path. It is this phase that ultimately leads to the appearance of the macroscopic anomalous Hall conductivity. Note that if the mean spin splitting field $h$ is taken to zero, $\sum_{\alpha \beta \gamma} \Im\left(t_{i \alpha j \beta} t_{j \beta k \gamma} t_{k \gamma i \alpha}\right)$ vanishes identically for any triad $(i, j, k)$. It is the presence of a macroscopic magnetization in the sample, or, in other words, broken time reversal symmetry, that results in a nonzero contribution from this term. Another important property of the third-order term in the current expectation value is that it leads to the appearance of a current between the sites $i$ and $j$, that is driven not by the potential difference on the bond $(i j)$, but by the potential differences on bonds $(i k)$ and $(j k)$. This property implies that the current, proportional to $F_{i j k}$, is on average transverse to the direction of the local electric field, as one would expect for the Hall current.

Eq.(6) reveals the microscopic origin of the AHE in the system. To obtain the anomalous Hall conductivity of a macroscopic sample, one needs to find a certain characteristic microscopic triad that determines the macroscopic conductivity. Since the distributions of both $G_{i j}$ and $F_{i j k}$ are very broad due to their exponential dependence on the random on-site potentials and Mn acceptor positions, the characteristic triad can not be obtained by a simple averaging over all possible triads. Instead, one needs to find a relationship between this generalized hopping conduction problem and a certain percolation problem, exactly like in the case of usual hopping conduction [17, 18]. It is, however, not an easy task, due 


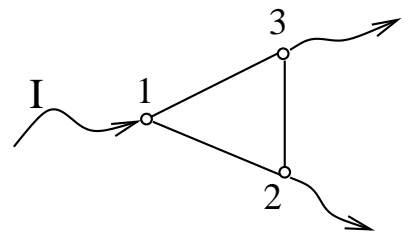

FIG. 1: A backbone node with an associated triad. $I$ is the current flowing in a macrobond terminating at site 1 in response to the external electric field $E$.

to a tensor nature of the transport brought about by the second term in Eq.(6). We believe that the best approach to this problem is the one used in Refs. 19, 20, 21] to investigate the problem of ordinary Hall effect in hopping conduction regime. This approach starts from the assumption that the problem can be solved perturbatively in the second, Hall term in Eq. (6). This is certainly a valid assumption if one wants to find the Hall conductivity to first order in the magnetic field (or magnetization in our case). Thus one imagines the following two-step iterative procedure. Initially, the second term in (6) is put to zero and external electric field is applied to the network. One then solves Kirchhoff's equations

$$
\sum_{j} G_{i j} V_{i j}^{0}=0
$$

and finds voltages $V_{i}^{0}$ at all nodes. The external electric field is then turned off and the zeroth-order voltages are substituted in the Hall term of Eq.(6). The Kirchhoff's equations at the second iteration take the following form:

$$
\sum_{j} G_{i j} V_{i j}=J_{i}^{H}
$$

where

$$
J_{i}^{H}=-\sum_{j k} F_{i j k}\left(V_{i k}^{0}+V_{j k}^{0}\right),
$$

i.e. now we have a network with external Hall currents $J_{i}^{H}$ flowing into every node. Thus the paradigm of the hopping Hall conduction in the approach we adopt is a random resistor network with external Hall currents 19.

To actually obtain explicit analytical results using the above procedure, one needs to know the distribution of Hall currents $J_{i}^{H}$ in the network. We will use ideas from the percolation theory picture of hopping transport [17, 18, 22] to find this distribution. According to this picture, one can assume that all the hopping current in the network is carried by a percolating cluster of large conductances, such that for all conductances in the cluster $G_{i j} \geq G_{c}$, where $\ln G_{c} \sim-\left(T_{0} / T\right)^{1 / 4}$ (we will limit our discussion to Mott's variable-range-hopping type conduction, but our theory, with minor modifications, is applicable to other kinds of hopping transport as well). The longitudinal conductivity of the network is then given by $\sigma_{x x} \approx G_{c} / L$, where $L$ is the correlation length of the cluster. The correlation length depends on temperature only as a power law 22] and we will not calculate it explicitly. Following Refs. [19, 20, 21], we now assert that the Hall conductivity in the system is associated exclusively with the current carrying backbone of the percolating cluster. The backbone can be visualized as a supernetwork of macrobonds and nodes, with typically three macrobonds intersecting at a node. The average distance between the nodes is $L$. Macrobonds can be assumed to be simply one-dimensional chains of conductances 22]. It is then clear that the exponentially dominant Hall current sources will be located at the nodes of the percolating cluster backbone. Since the nodes are far away from each other, we may regard them as independent sources of the Hall emf. Thus the problem reduces to finding the Hall emf developed at a single isolated node of the backbone in response to the current flowing through the node 19, 20, 21. Applying the above described two-step iterative procedure to a single node with an associated triad of sites shown in Fig 1 we obtain:

$$
\begin{aligned}
V^{H} \equiv V_{32} & =\frac{G_{13} J_{2}^{H}-G_{12} J_{3}^{H}}{G_{13} G_{32}+G_{12} G_{23}+G_{31} G_{12}} \\
& =\frac{I F_{123}}{G_{13} G_{32}+G_{12} G_{23}+G_{31} G_{12}},
\end{aligned}
$$

where $V^{H}$ is the Hall emf, $I$ is the current flowing through the node, and we have neglected unimportant numerical prefactors. Since the average distance between the nodes is $L$, the macroscopic Hall field in the sample is given by:

$$
E^{H}=I L^{-1}\left\langle\frac{S_{123} F_{123}}{G_{13} G_{32}+G_{12} G_{23}+G_{31} G_{12}}\right\rangle_{c},
$$

where $\langle\ldots\rangle_{c}$ denotes averaging over the on-site energies and intersite distances in the triad $(1,2,3)$, such that the condition

$$
2 r_{i j} / \xi+\epsilon_{i j} / T \leq\left(T_{0} / T\right)^{1 / 4}
$$

is satisfied for every bond $(i j)$ in the triad [18] and $S_{123}=\operatorname{sgn}\left[\mathbf{h} \cdot\left(\mathbf{r}_{12} \times \mathbf{r}_{13}\right)\right]$ accounts for the orientation of the triangle with respect to $\mathbf{h}$. Taking into account that the Hall current density is given by $j^{H}=\sigma_{x x} E^{H}$ and $I=\sigma_{x x} L^{2} E$, we obtain the following expression for the anomalous Hall conductivity [19, 20]:

$$
\sigma_{x y}^{A H}=L \sigma_{x x}^{2}\left\langle\frac{S_{123} F_{123}}{G_{13} G_{32}+G_{12} G_{23}+G_{31} G_{12}}\right\rangle_{c} .
$$

Let us write down the averaging explicitly as an integral over intersite distances and on-site energies:

$$
\begin{aligned}
& \sigma_{x y}^{A H}=L \sigma_{x x}^{2} \frac{1}{\mathcal{N}} \int_{c} d \mathbf{r}_{12} d \mathbf{r}_{23} d \mathbf{r}_{31} \int_{c} d \epsilon_{1} d \epsilon_{2} d \epsilon_{3} \\
& \times \varrho\left(\epsilon_{1}\right) \varrho\left(\epsilon_{2}\right) \varrho\left(\epsilon_{3}\right) \frac{S_{123} F_{123}}{G_{13} G_{32}+G_{12} G_{23}+G_{31} G_{12}},(17)
\end{aligned}
$$


where $\mathcal{N}$ is a normalization factor. The densities of states $\varrho(\epsilon)$ can be expanded around the Fermi energy $\varrho(\epsilon) \approx \varrho_{0}+\frac{d \varrho_{0}}{d \epsilon} \epsilon$, since hopping is restricted to a narrow interval of order $T\left(T_{0} / T\right)^{1 / 4}$ around the Fermi energy. Substituting the expanded densities of states in Eq.(17), one finds that the zeroth order term, proportional to $\varrho_{0}^{3}$, vanishes. The reason is that $F_{123}$ changes sign under the inversion of all the on-site energies, while the bond conductances $G_{i j}$ are invariant under this transformation. Thus we obtain:

$$
\sigma_{x y}^{A H}=L \sigma_{x x}^{2} \frac{d \ln \varrho_{0}}{d \epsilon}\left\langle\frac{S_{123} F_{123}\left(\epsilon_{1}+\epsilon_{2}+\epsilon_{3}\right)}{G_{13} G_{32}+G_{12} G_{23}+G_{31} G_{12}}\right\rangle_{c},
$$

where the average is now over a uniform distribution of the on-site energies. Approximating the average of the quantity inside the angular brackets by its maximum value and expanding to first order in $h$, one finally obtains the following expression for the anomalous Hall hopping conductivity:

$$
\sigma_{x y}^{A H} \sim L \sigma_{x x}^{2} \frac{d \ln \varrho_{0}}{d \epsilon} \frac{h T}{e^{2} t_{3 / 2}}\left(T_{0} / T\right)^{1 / 4} e^{-\left(T_{0} / T\right)^{1 / 4}},
$$

where we have used the property that $t_{3 / 2} \gg t_{1 / 2}$ in GaMnAs [13]. Analogous calculation in the case of the ordinary Hall effect gives

$$
\sigma_{x y}^{O H} \sim L \sigma_{x x}^{2} \frac{B \xi^{2} T}{\operatorname{cet}_{3 / 2}} e^{-\left(T_{0} / T\right)^{1 / 4}} .
$$

Thus, the ratio of the ordinary and anomalous Hall conductivities is

$$
\frac{\sigma_{x y}^{A H}}{\sigma_{x y}^{O H}} \sim h \frac{d \ln \varrho_{0}}{d \epsilon} \frac{c}{e B \xi^{2}}\left(T_{0} / T\right)^{1 / 4} .
$$

Several concrete experimentally testable predictions follow from the above equations. First, according to (19), $\sigma_{x y}^{A H}$ is proportional to the derivative of the density of states at the Fermi energy. This means that the anomalous Hall conductivity can be expected to change sign as the Fermi level crosses the density-of-states maximum in the impurity band. This is in contrast to the ordinary Hall conductivity $\sigma_{x y}^{O H}$ which has the same sign everywhere in the impurity band. Thus AHE can potentially be a very useful tool in elucidating the character of hole carriers in (Ga,Mn)As. The second prediction concerns the temperature dependence of $\sigma_{x y}^{A H}$. As seen from Eq. (19), the leading temperature dependence of the anomalous Hall conductivity is $\sigma_{x y}^{A H} \propto \exp \left[-\left(T_{0} / T\right)^{1 / 4}\right]$, i.e. it depends on temperature in the same manner as the longitudinal conductivity $\sigma_{x x}$. The same is true for the ordinary Hall conductivity. This, in particular, means that the Hall resistivity $\varrho_{x y} \sim \varrho_{x x}$ as a function of temperature, i.e. it diverges as $T \rightarrow 0$. Thus our calculation is consistent with the view [14] that the transverse resistivity of the "Hall insulator" diverges at $T \rightarrow 0$.
In conclusion we would like to point out that our theory seems to be consistent with recent magnetotransport experiments on digitally-doped GaMnAs samples [23].

We are grateful for useful discussions with W. Allen, E. Gwinn, G. Fiete and E. Johnston-Halperin. We would especially like to thank D.D. Awschalom for his encouragement and support. This work was supported by DARPA/ONR N00014-99-1-1096, by the NSF under grant DMR-9985255 and by the Sloan and Packard foundations.

[1] S.J. Potashnik et al., Appl. Phys. Lett. 79, 1495 (2001).

[2] J. König et al., in Electronic Structure and Magnetism of Complex Materials, edited by D.J. Singh and D.A. Papaconstantopoulos (Springer Verlag, 2002); T. Jungwirth et al., Phys. Rev. B 59, 9818 (1999); T. Dietl et al., Science 287, 1019 (2000); J. König et al., Phys. Rev. Lett. 84, 5628 (2000).

[3] A. Van Esch et al., Phys. Rev. B 56, 13103 (1997); J. Okabayashi et al., Phys. Rev. B 64, 125304 (2001); M. Berciu and R.N. Bhatt, Phys. Rev. Lett. 87, 107203 (2001); A. Kaminski and S. Das Sarma, Phys. Rev. Lett. 88, 247202 (2002).

[4] J. Smit, Physica (Amsterdam) 21, 877 (1955); ibid. 24, 39 (1958).

[5] J.M. Luttinger, Phys. Rev. 112, 739 (1958).

[6] L. Berger, Phys. Rev. B 2, 4559 (1970).

[7] H. Ohno et al., Phys. Rev. Lett. 68, 2664 (1992); H. Ohno et al., Appl. Phys. Lett. 69, 363 (1996); H. Ohno, J. Magn. Magn. Mater. 200, 110 (1999); H.X. Tang et al., Phys. Rev. Lett. 90, 107201 (2003).

[8] T. Jungwirth et al., Phys. Rev. Lett. 88, 207208 (2002); Appl. Phys. Lett. 83, 320 (2003).

[9] J. Ye et al., Phys. Rev. Lett. 83, 3737 (1999).

[10] S.H. Chun et al., Phys. Rev. Lett. 84, 757 (2000); Y. Lyanda-Geller et al., Phys. Rev. B 63, 184426 (2001).

[11] R. Shindou et al., Phys. Rev. Lett. 87, 116801 (2001); Y. Taguchi et al., Science 291, 2573 (2001); M. Onoda and N. Nagaosa, J. Phys. Soc. Jap. 71, 19 (2002).

[12] T. Holstein, Phys. Rev. 124, 1329 (1961).

[13] G.A. Fiete et al., cond-mat/0212074 [Phys. Rev. Lett. (to be published)].

[14] O. Entin-Wohlman et al., Phys. Rev. B 51, 11584 (1995); Phys. Rev. Lett. 75, 4094 (1995).

[15] Y. Galperin and O. Entin-Wohlman, Phys. Rev. B 54, 9346 (1996).

[16] A. Miller and E. Abrahams, Phys. Rev. 120, 745 (1960).

[17] B.I. Shklovskii and A.L. Efros, Zh. Eksp. Teor. Fiz. 60, 867 (1971) [Sov. Phys. JETP 33, 468 (1971)].

[18] V. Ambegaokar et al., Phys. Rev. B 4, 2612 (1971).

[19] H. Böttger and V.V. Bryksin, Phys. Stat. Sol. B 81, 433 (1977).

[20] L. Friedman and M. Pollak, Philos. Mag. B 38, 173 (1978); ibid. 44, 487 (1981).

[21] Y.M. Galperin et al., Zh. Eksp. Teor. Fiz. 99, 343 (1991) [Sov. Phys. JETP 72, 193 (1991)].

[22] A.S. Skal and B.I. Shklovskii, Fiz. Tekh. Poluprovodn. 8, 1586 (1974) [Sov. Phys. Semicond. 8, 1029 (1974)].

[23] W. Allen, T.C. Kreutz and E.G. Gwinn (to be published). 